\title{
Evaluation of Accidents and Incidents of Injury Registered in Medical Centers Affiliated to Shahid Beheshti University of Medical Sciences
}

\author{
Mohammad Abdolvand*, Shahnam Arshi, Mohammad Reza Sarbazi \\ Deputy of Health, Shahid Beheshti University of Medical Sciences, Tehran, Iran
}

\section{A BSTRACT}

Introduction: The purpose of this study was to investigate injuries resulting from accidents and incidents referred to medical centers and hospitals of Shahid Beheshti University of Medical Sciences. Materials and Methods: This study is a cross-sectional study. The data of all injured patients admitted to hospitals of Shahid Beheshti University of Medical Sciences from March 2014 to March 2015 were reviewed. Accidents and injuries were categorized according to the tenth version of international classification of diseases. Data collected by means of forms and software injury register by Hospital emergency department staff to interview and hospital records, were completed. Results: In total, 131967 cases were reviewed,in which $66 \%$ were male and $34 \%$ were female. Traumas, traffic accidents, and falls were major causes of accidents and injuries. Accidents and injuries were more prevalent in 2029 years old (the younger age groups; $51 \%$ ). Individuals living in urban areas sustained more injuries compared to individuals from rural areas. A total of 194 deaths were reported due to accidents and injuries. Conclusion: Our findings indicated that traffic accidents constitute a large proportion of accident and injuries. Productive groups (young male) were the most victims of accidents and injuries. Training of different groups, including before and during recruitment, training mothers, and elderly seems to be beneficial. Further studies to improve the registration tools for a better access to reliable data are needed. Promotion knowledge and training, especially in the younger age group, intervention to resolve the accident prone spots as well as technical defects are recommended to reduce the traffic accidents.

\section{Key words:}

1. Vital Statistics

2. Accidents

3. injuries

*Corresponding Author: Mohammad Abdolvand

E-mail: m.Abdolvand@gmail.com 


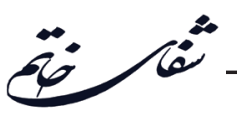

بررسى مصدومين سوانح و حوادث ثبت شده در مراكز درمانى وابسته به دانشگاه علوم يزشكى شهيد

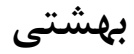

\author{
محمد عبدالوند"، شهنام عرشى، محمد رضا سربازى \\ معاونت امور بهداشتى، دانشكاه علوم يزشكى شهيد بهشتى، تهران، ايران
}

كليد وازهها:

مقدمه: هدف از اين مطالعه بررسى صدمات ناشى از تصادفات و حوادث مراجعه كننده به مراكز درمانى و

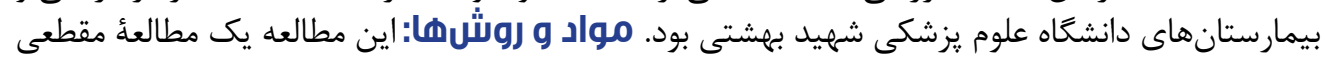

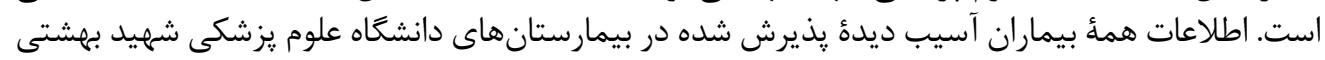

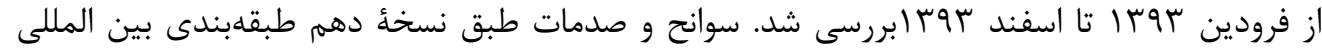

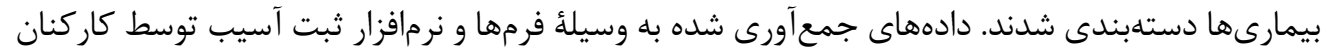

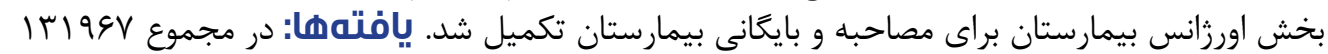

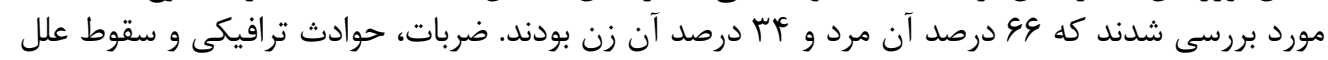

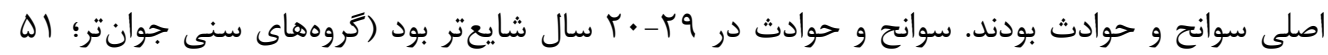

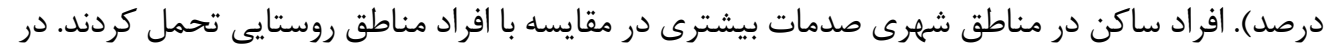

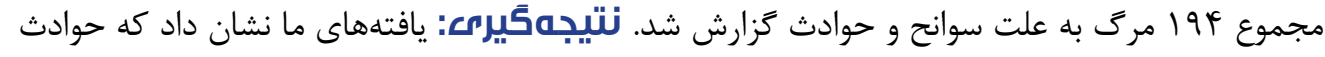

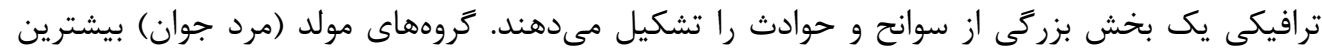

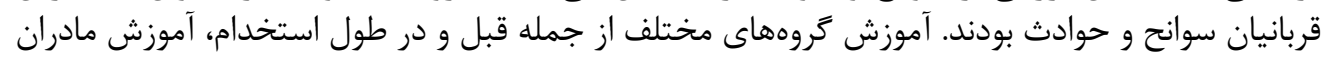

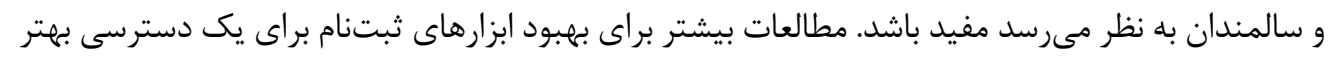

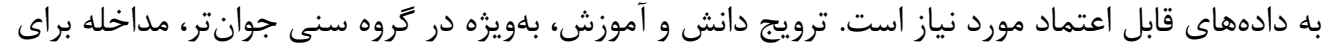

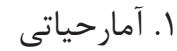

ب.

r. مصدوميتها

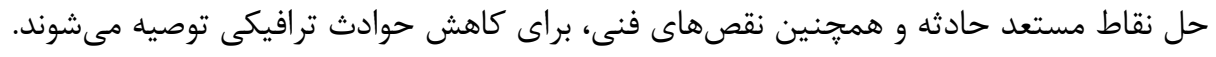

" نويسنده مسئول: محمدعبدالوند آدرس الكترونيكى: m.Abdolvand@gmail.com 


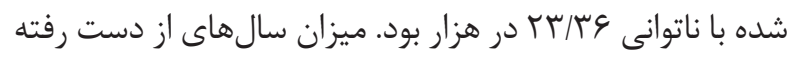

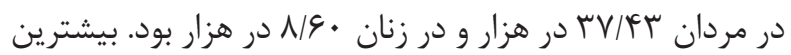

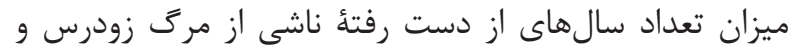

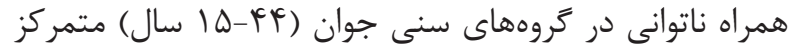

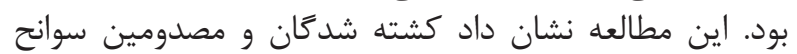

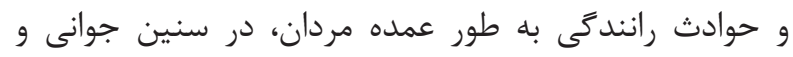

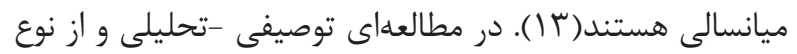

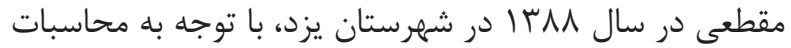

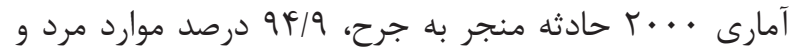

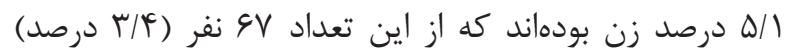

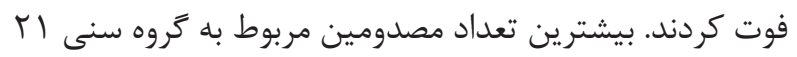

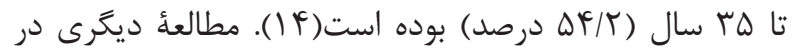

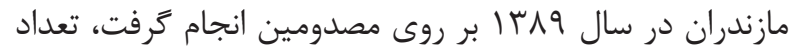

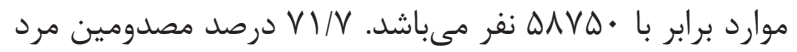

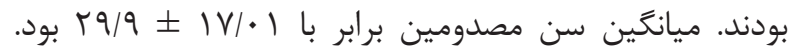

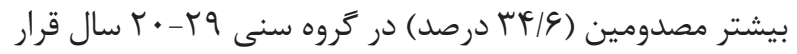

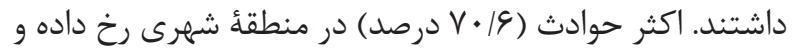

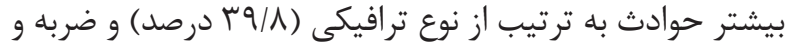

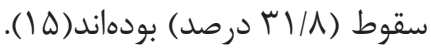

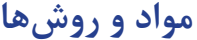

مطالعهُ حاضر يك مطالعؤ مقطعى است كه به به بروسى وضع وضعيت

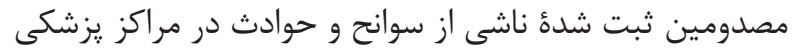

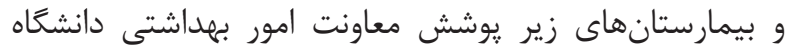

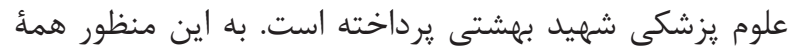

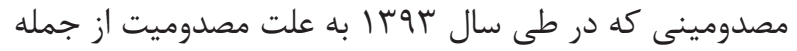

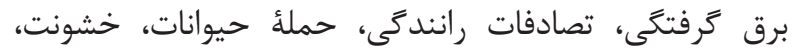

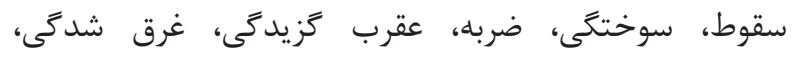

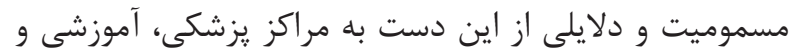

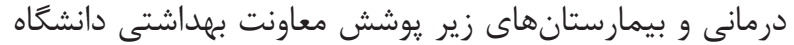

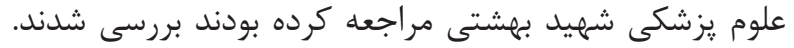

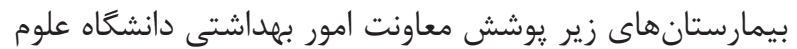

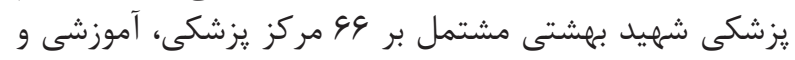
درمانى و بيمار ستان مى بـاشد.

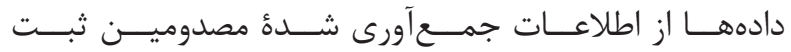

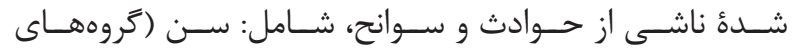

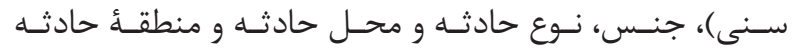

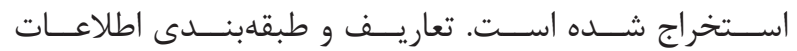

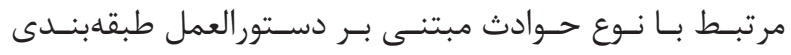

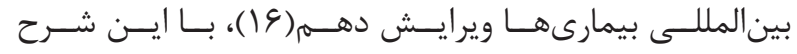

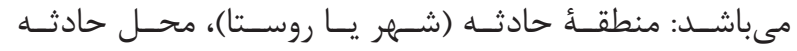

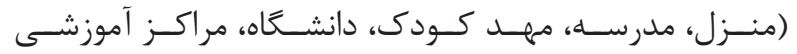

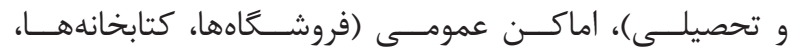

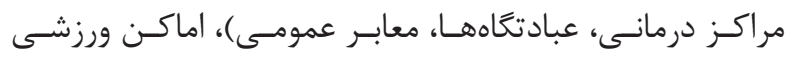

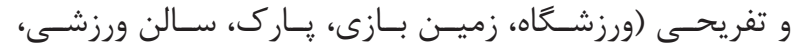

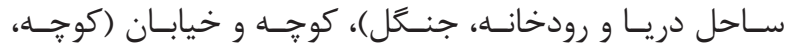

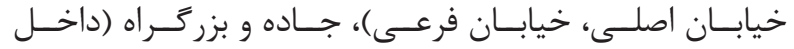

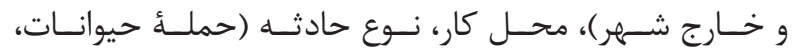

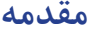

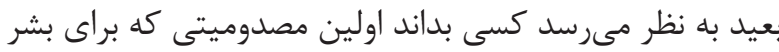

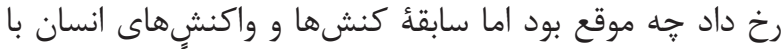

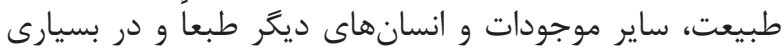

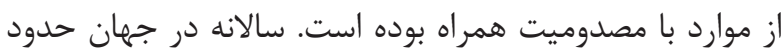

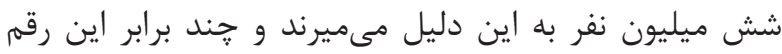

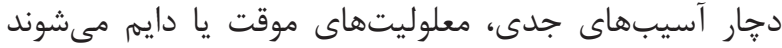

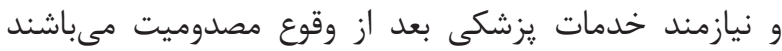

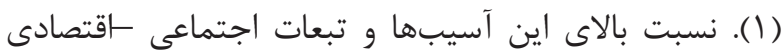

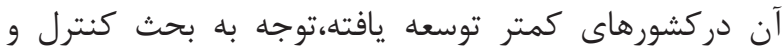

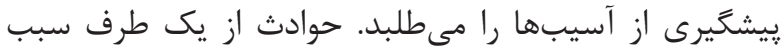

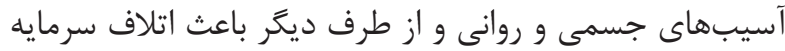

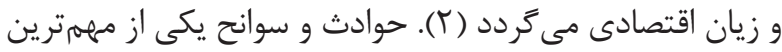

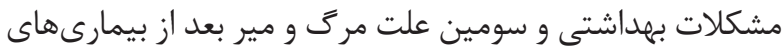

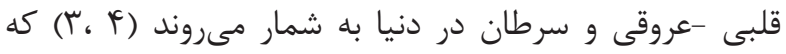

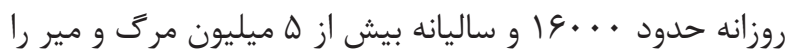

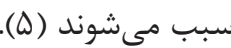

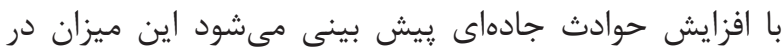

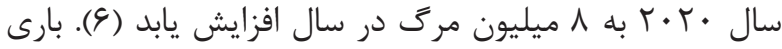

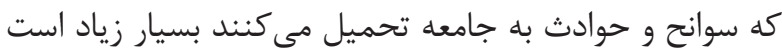

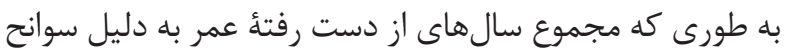

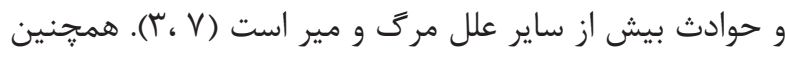

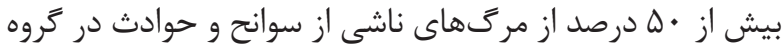

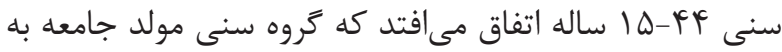

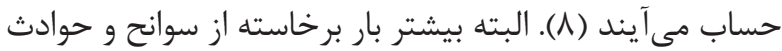

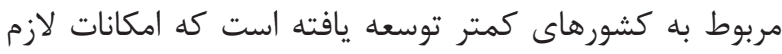

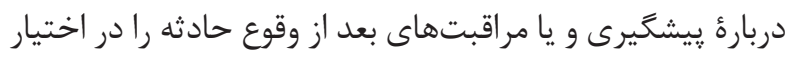

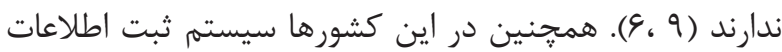

سوانح و حوادث دقيق و قابل اعتمادى وجود ندارد (• (1).

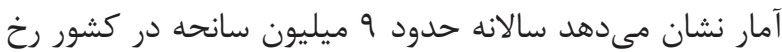

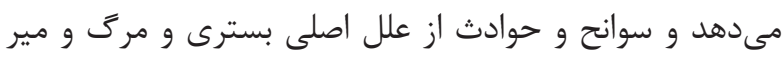

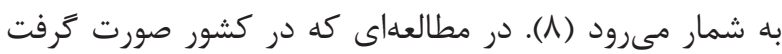

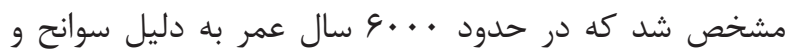
حوادث از دست رفته است (^). شايعترين نوع دور سوانح در در ايران

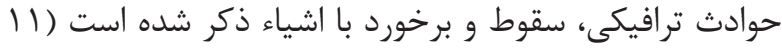

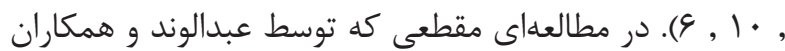

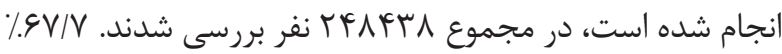

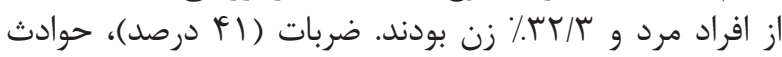

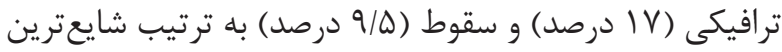

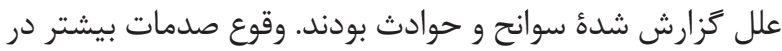

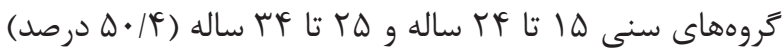

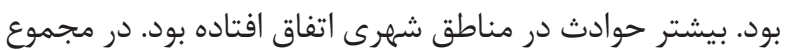

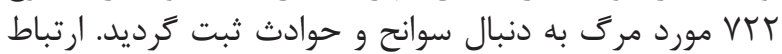

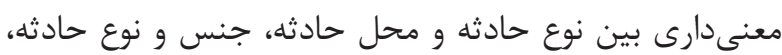

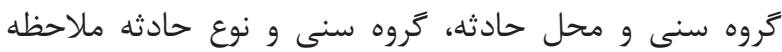

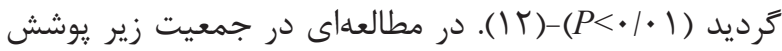

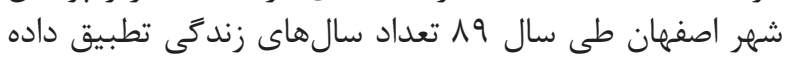




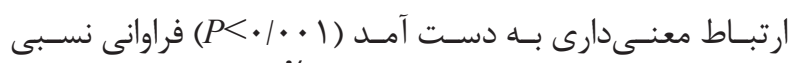

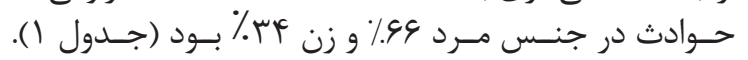

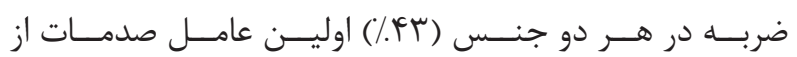

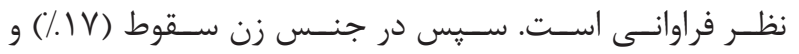

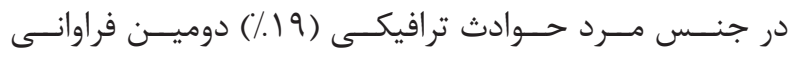

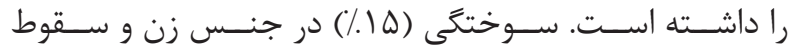

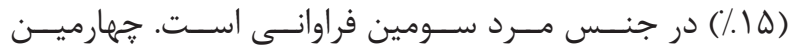

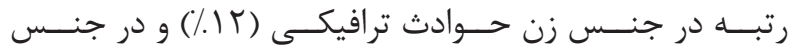

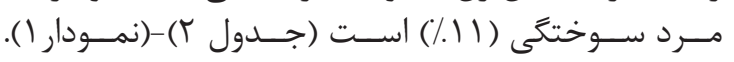

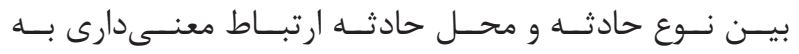

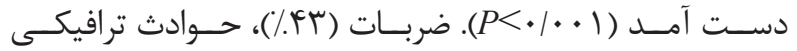

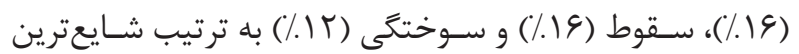

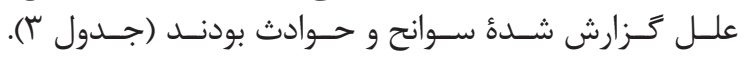

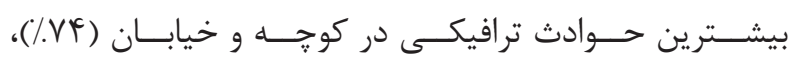

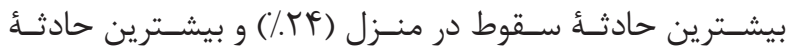

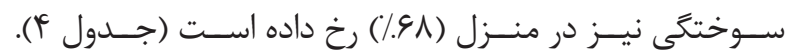

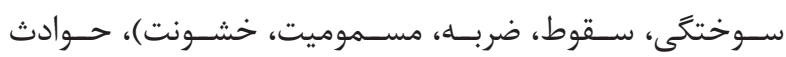

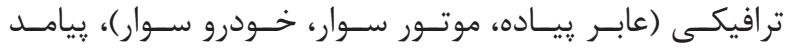

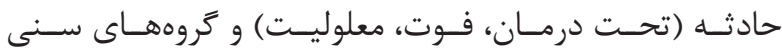

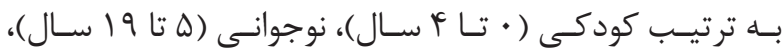

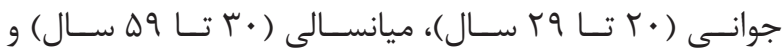

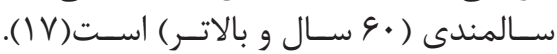

تجزيه وتحليل دادهها

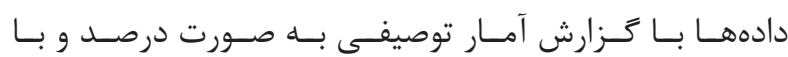

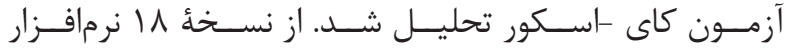

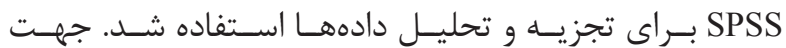

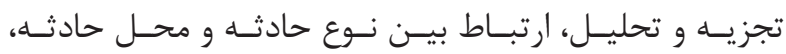

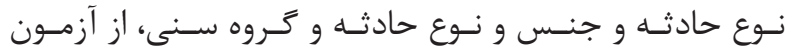

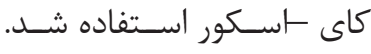

يافتهها

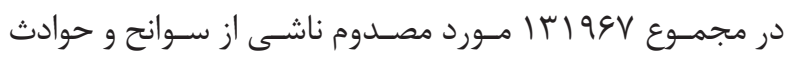

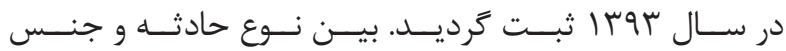

\begin{tabular}{|c|c|c|c|c|c|c|c|c|c|c|c|c|c|}
\hline$?$ & $\begin{array}{l}\{ \\
\xi \\
\xi \\
\xi\end{array}$ & 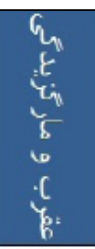 & $\dot{\gamma}$. & G. & $\begin{array}{l}b \\
6 \\
6\end{array}$ & $\begin{array}{l}\stackrel{c}{\breve{s}} \\
\dot{y} \\
\frac{y}{\varepsilon}\end{array}$ & $\xi$ & $\begin{array}{l}\frac{c}{\xi} \\
\frac{\xi}{6} \\
\xi \\
\xi\end{array}$ & 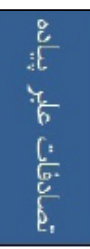 & 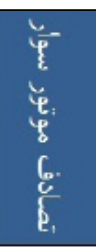 & 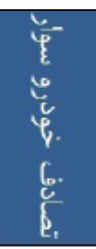 & 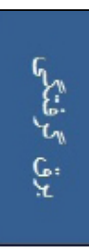 & $\begin{array}{l}\varepsilon_{3} \\
\bar{q} \\
c \\
\varepsilon \\
\varepsilon \\
\xi\end{array}$ \\
\hline$F D I \& Y$ & 1189 & $I Y \Lambda$ & 19400 & 99.9 & VAVI & TVTI & $v f q$ & $1 \cdot 0$ & $r \cdot \Lambda f$ & AVV & $r \cdot Y Q$ & $1 \cdot f$ & زن \\
\hline$\%$ HF & $\% . \Delta Y$ & $\%$ & $\%$ YrF & $\%$ Fr & $\%$ & $\%$ \%q & $\% 19$ & $\%$ \% & r & $\% 11$ & ry & Y.TF & رصد \\
\hline$\wedge \& \Lambda \cdots$ & $1 \cdot 18$ & TYG & YVAYI & QfYr & IKEIY & $\Delta Q Y T$ & & ris & FYTQ & $Y F Y D$ & frel & גזr & مرد \\
\hline 7.94 & $\%$ \& & 7.94 & 7.94 & $\% \Delta \Lambda$ & $7.9 Y$ & 7.91 & 7.11 & $\% \vee v$ & $7.9 \mathrm{~V}$ & $\% 19$ & 7.51 & $\%$ VG & درصد \\
\hline $14194 \mathrm{~V}$ & rted & rof & DqqVG & TETrT & r.fAF & १९६४ & एव人 & Frt & दा. 9 & $\Lambda r \cdot r$ & gfre & fFY & co \\
\hline $\begin{array}{l}\% \\
1 \%\end{array}$ & $\% 1 \ldots$ & $\% 1 \ldots$ & $\% 1 \ldots$ & $\% 1 \ldots$ & $\% 1 \ldots$ & $\% 1 \ldots$ & $\% 1 \ldots$ & $\% 1 \ldots$ & $\% 1 \ldots$ & $\% 1 \ldots$ & $\% 1 \ldots$ & $\%$ & ص \\
\hline
\end{tabular}

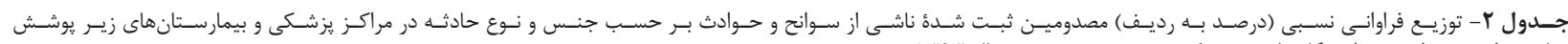

\begin{tabular}{|c|c|c|c|c|c|c|c|c|c|c|c|c|c|c|}
\hline \multirow[b]{2}{*}{$\xi$} & \multirow[b]{2}{*}{ 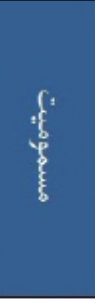 } & \multirow[b]{2}{*}{$\begin{array}{l}\xi_{h} \\
\xi \\
c_{0} \\
c_{0} .\end{array}$} & \multirow[b]{2}{*}{$\begin{array}{l}q_{n} \\
\varepsilon \\
c \\
\vdots \\
\vdots \\
c \\
c \\
k \\
k=\end{array}$} & \multirow[b]{2}{*}{$\xi$} & \multirow[b]{2}{*}{$\xi$} & \multirow[b]{2}{*}{$\begin{array}{l}\sigma \\
\sigma_{0}\end{array}$} & \multirow[b]{2}{*}{$\begin{array}{l}\frac{c}{\breve{g}} \\
\frac{\tilde{G}}{\varepsilon}\end{array}$} & \multirow[b]{2}{*}{$\frac{\xi}{4}$} & \multirow[b]{2}{*}{$\begin{array}{l}\frac{\xi}{E} \\
\frac{\xi}{q} \\
\xi \\
\xi\end{array}$} & \multicolumn{3}{|c|}{ حوادث ترافيكى } & \multirow[b]{2}{*}{$\begin{array}{l}\xi_{n} \\
\xi_{0} \\
\xi_{n} \\
c_{0}\end{array}$} & \multirow[b]{2}{*}{$\xi$} \\
\hline & & & & & & & & & & 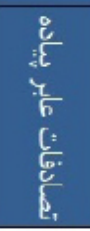 & 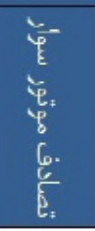 & 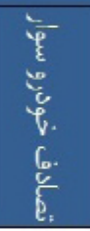 & & \\
\hline FOISV & 1189 & 19 & IrA & $194 \Delta \Delta$ & $9 q . q$ & vivi & ו ו"r & $v \notin q$ & $1 \cdot \Delta$ & $r \cdot \Lambda F$ & AVV & $r \cdot v \Delta$ & 1.4 & زن \\
\hline$\% 1 \ldots$ & $\%$ & $\%$ & $\% \cdot / \pi$ & $\% \mu r$ & $\% 10$ & $\% 1 \mathrm{~V}$ & $\%$ & $\% r$ & $\% \cdot \pi$ & $\%$ & $\% r$ & $\%$ & $\% \cdot \pi$ & درصد \\
\hline 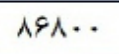 & $1 \cdot 19$ & rV & TKE & सVGYI & afru & Irदाז & DQTT & זשrז & אוא & Frts & VFro & स्दा & גשץ" & مرد \\
\hline$\% 1 \ldots$ & $\% 1$ & $\%$ & $\%$ & $\%$ FT & $\% 11$ & $\% 10$ & $\% V$ & $\% x$ & $\% \cdot / f$ & $\%$ & $\% 9$ & $\% .0$ & $\% \cdot / f$ & درصد \\
\hline $1 \% 199 \mathrm{~V}$ & TKGO & $\Delta \varepsilon$ & rof & Dgqve & TאוFT & t.FAT & १९६ए & rasi & Frt & दr.q & $\Lambda T \cdot r$ & gers & fer & \\
\hline$\% 1 \cdots$ & $\%$ & $\%$ & $\%$ & $\%$ & $\% 14$ & $\% 18$ & $\% \vee$ & $\%$ & $\%$ & $\%$ & $\% 9$ & $\%$ & $\%$ & 20 \\
\hline
\end{tabular}




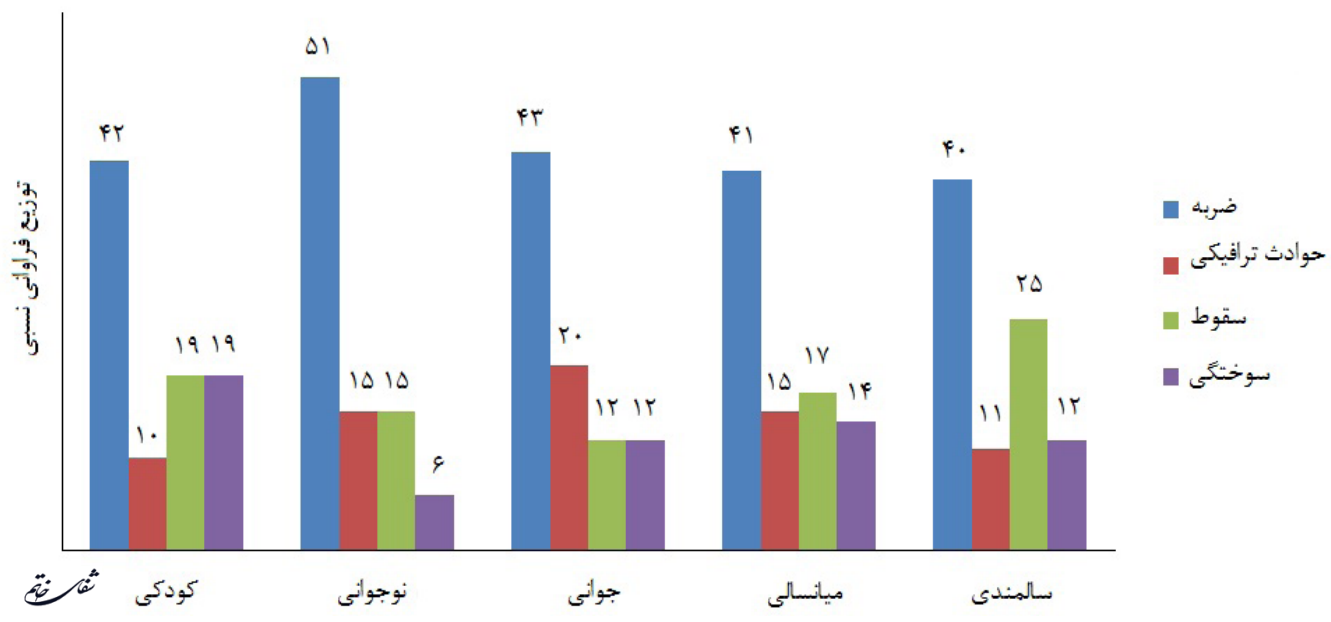

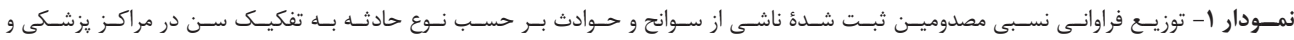

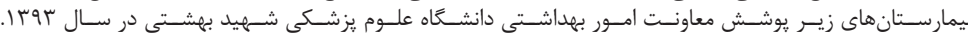

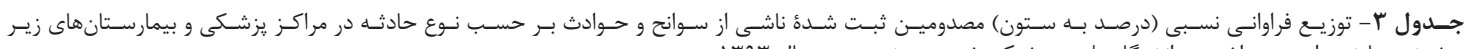

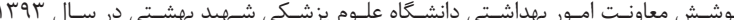

\begin{tabular}{|c|c|c|c|c|c|c|c|c|c|c|}
\hline جa & نامعلوم & منزل & مدرسهة و آماكي & معل كار & كوجه و خيلان & موارد & بزركراهو & و تفريتى ورزئ & عموعي & 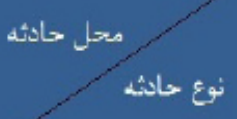 \\
\hline$\%$ & $\%$ & $\% 1$ & $\%$ & $\% 1$ & $\%$ & $\%$ & $\%$ & $\%$ & $\%$ & برق گرفتخى \\
\hline$\% 19$ & 15 & $\%$ & $\%$ & $\%$ & $\% T$ & $\%$ & YAF & $\%$ & $\%$ & ترافيك (خودرو، موتور، \\
\hline$\%$ & $\%$ & $\% 1$ & $\%$ & $\%$ & $\%$ & $\% 1$ & $\%$ & $\% \cdot$ & $\% 1$ & حهلة حيولاتات \\
\hline $7 / r$ & $\% r$ & $\%$ & $\% T$ & $\% 1$ & $\%$ & $\% 1$ & $\% 1$ & $\% 1$ & $\% 11$ & خشونت \\
\hline$\% \gamma$ & $\% 11$ & $\% \Lambda$ & $\%$ & $\%$ & $7 / 1$ & $\% \Delta r$ & $\% 1$ & $\pi / r$ & $\%$ & ماير \\
\hline$\% 18$ & 7.54 & $\% 1 r$ & $\% 10$ & $\% 1 \mathrm{~V}$ & $\% 9$ & 19 & $\pi / T$ & 719 & $\% 10$ & سقوط \\
\hline 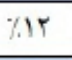 & $\%$ & $\pi / 19$ & $/ \Delta$ & $\% 19$ & $\%$ & $\% \Delta$ & $\% \pi$ & $\gamma / \mathrm{V}$ & $\% \pi$ & سوختكى \\
\hline $7+4$ & $7 \% 4$ & YFT & $\% \mathrm{YT}$ & $/ \Delta F$ & $\%$ & 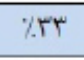 & $\% 1 / 1$ & $\%$. & 7.91 & ضردية \\
\hline$\%$ & $\%$ & $\% 1$ & $\%$ & $\%$ & $\%$ & $\%$ & $\%$ & $\%$ & $\%$ & عقرب و مارگزيدَّى \\
\hline$\%$ & $\%$ & $\% \Delta$ & $2 / 4$ & $\% 1$ & $\%$ & $\% 1$ & $\%$ & $\%$ & $\pi / 1$ & مسموميت \\
\hline ثم & $\% 1+$ & $\% 1 \cdots$ & $\% 1 \ldots$ & $\% 1$. & $\%$ & $\% 1+$ & $\% \cdots$ & $\% 1$. & $\% 1$. & جهو \\
\hline
\end{tabular}

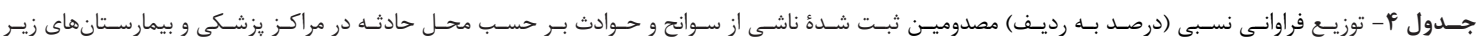

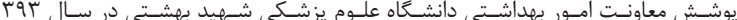

\begin{tabular}{|c|c|c|c|c|c|c|c|c|c|c|}
\hline كل & ن امعلوم & | منزل & مدرسه و اماكن & مجل كار & خويابه و & ساير موارد & بزركراه و جاده & و تفريحى ورزثى & مامومى & نو حاثنه حسل حاثث \\
\hline$\%$. & $\% \mu$ & 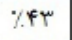 & $\% 1$ & $\%$ \% & $\% 11$ & $\% 1$ & $\%$ & $\% \mu$ & $\% v$ & برق كرفتكى \\
\hline$\% \ldots$ & $\%$ & $\% \Delta T$ & $\%$ & $\% . \Delta$ & $\% \pi$ & $\%$ & $\% 1$ & $\% \pi$ & $\% 1$. & حمله حيوانات \\
\hline$\% 1$. & $\%$ & $\% 19$ & $\% 1$ & $\% f$ & $7 f f$ & $\% 1$ & $\% 1$ & $\pi T$ & $\% 11$ & خشونت \\
\hline$\% 1$. & $\% / \mu$ & $\%$ HF & $\% 1$ & $\% 1$. & $\% 19$ & $\% 1$ & $\% \cdot$ & $1 / 9$ & $\%$ & ستوط \\
\hline$\% 1$. & $\%$ & $\%, \mathrm{~A}_{1}$ & $\%$ & $\% 1 \%$ & $\gamma, \Lambda$ & $\% 1$ & $\% \cdot$ & $\% r$ & $\% 1$ & سوختعى \\
\hline$\% 1$. & $\% 1 \mathrm{~V}$ & $\% / r \Lambda$ & $\%$ & $\% 11$ & $\%$ /rf & $\%$ & $\% 1$ & $\% / 1$ & $\% v$ & ضربد \\
\hline$\% \cdots$ & $\%$ & $\%, 90$ & $\%$ & $\% 14$ & $\% r$ & $\% y$ & $\%$ & $\% T$ & 1.9 & عقرب و مارتزيدتى \\
\hline$\% 1$. & $\% r$ & $\%$. & $\% 1$ & $\% \Delta$ & $\% / r$ & $\%$ & $\%$ & $\% 1$ & $\%$ & مسموميت \\
\hline$\% \ldots$ & $\% v$ & $\%$ & $\%$ & $\% 1$ & $\% V^{4}$ & $\%$ & $\% 1 \mathrm{Y}$ & $\% \cdot$ & $\% 1$ & ترافيك \\
\hline$\% \cdots$ & $\% \mathrm{TV}$ & $\% \pi r$ & $\% 1$ & 7.5 & $\% 9$ & $\% 19$ & $\% \cdot$ & $\% 1$ & $\%$ & ساير \\
\hline $11 \ldots$ & $\% 11$ & $7 / \% q$ & $\% 1$ & 7.9 & $7 / r \lambda$ & $\%$ & $\% r$ & $\% \Delta$ & $1 / 0$ & كل \\
\hline
\end{tabular}




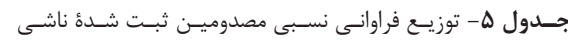

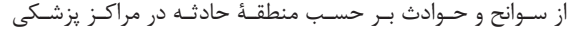

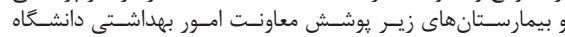

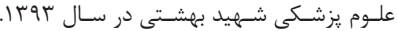

\begin{tabular}{|c|c|}
\hline درصد & منطقة حادثة \\
\hline$\%$ & خارج از شعر و روستا \\
\hline$\% 1 / \Delta$ & روستايى \\
\hline$\% 9 \Delta / r$ & شعرى \\
\hline$\%$ & نامعلوم \\
\hline$\% 1 \cdots$ & كل \\
\hline
\end{tabular}

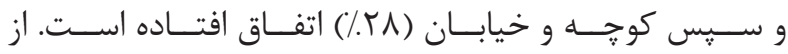

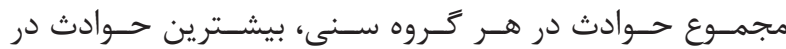

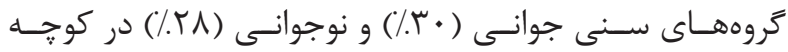

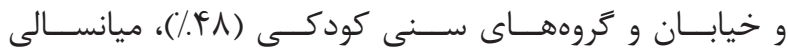

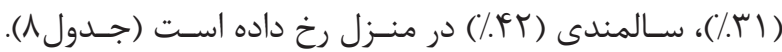

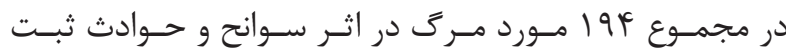

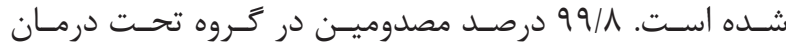

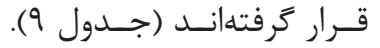

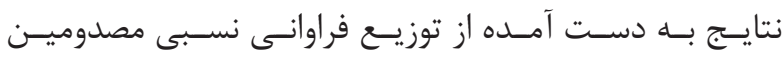

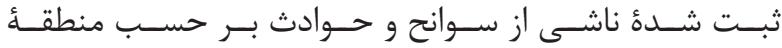

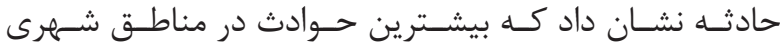

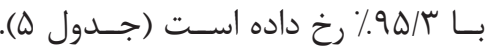

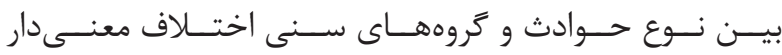

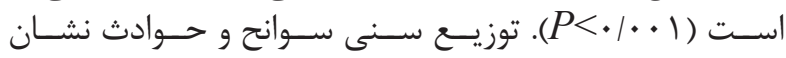

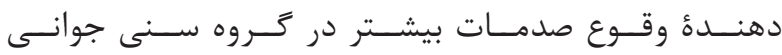

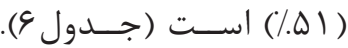

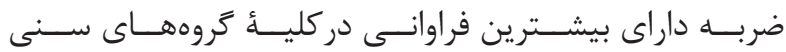

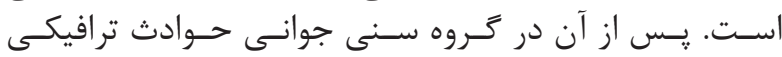

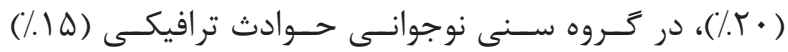

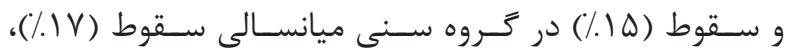

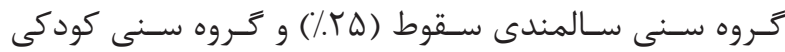

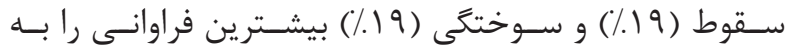

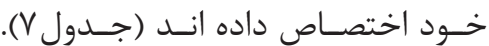

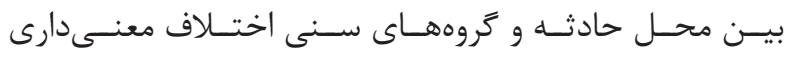

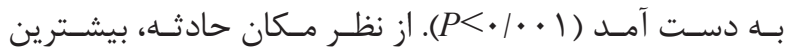

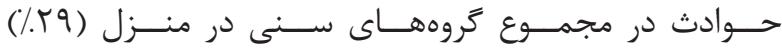

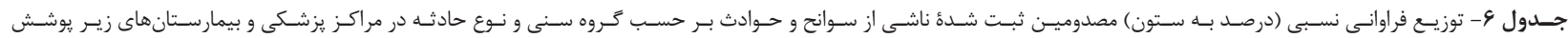

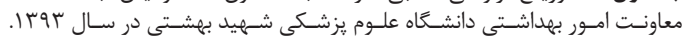

\begin{tabular}{|c|c|c|c|c|c|c|c|c|c|c|c|c|c|}
\hline \multirow[b]{2}{*}{ ج } & \multicolumn{12}{|c|}{ نوع حادث } & \multirow[b]{2}{*}{ كروبه سـن } \\
\hline & مسموهبيت & عارئزيد عثى و & ضربه & سوخيكى & ستوط & سابر موارد & خشونت & حملة حيوات & تصانياث & تصوتئ & خوثرو سوار & برق كرقتي & \\
\hline$\% 1 \%$ & $\% 1 \%$ & $\% 1$. & $7.1 \%$ & $\% 19$ & $\% 10$ & $\% 1 r$ & $\%$ & $\% 10$ & $\% 1 r$ & $\pi r$ & 次 & $1 / 9$ & كودكي \\
\hline$\% 1 r$ & $\% 1 r$ & $\% 1 r$ & 710 & $7 / 9$ & $\% 1 \%$ & $\% 11$ & $\% 15$ & $\% 14$ & $\% 1 r$ & $\% 14$ & $1 / 9$ & $\% 10$ & نوجرائى \\
\hline$\% / \Delta 1$ & $\% \Delta s$ & $\% \Delta r$ & $1 / \Delta 1$ & $\%$ & $\gamma / 41$ & $1 / p q$ & 1.99 & $\% \Delta$. & $\%$ & $\% / \mathrm{V}$. & s. & $\% \Delta \Delta$ & جوانى \\
\hline$\% 1 \%$ & $\% 1$. & $\% 1 \mathrm{~V}$ & $\% 1 \mathrm{~T}$ & $\% 18$ & $\% 18$ & $\% 18$ & $\% 11$ & $\% 1 r$ & $\% 1 \mathrm{~F}$ & 7.11 & $\% 1 \%$ & $\% 1 \%$ & هبانسلى \\
\hline$\%$ & $7 / 9$ & $\% / V$ & $1 / 9$ & $1 / 9$ & $\% 19$ & $\% 1 r$ & 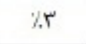 & $1 / 9$ & $\% 11$ & $\% \varphi$ & 㰡 & $\% Y$ & سالمندى \\
\hline 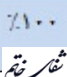 & $\% 1 \ldots$ & $\% 1 \cdots$ & $\pi 1 \cdots$ & $\pi 1 \cdots$ & $\% 1 \ldots$ & $\% 1 \cdots$ & $\% 1 \ldots$ & $\% \ldots$ & $\% 1 \ldots$ & $\% 1 \cdots$ & $\% 1 \cdots$ & $\% 1 \cdots$ & جم \\
\hline
\end{tabular}

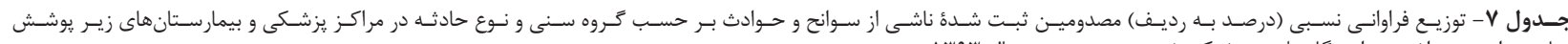

\begin{tabular}{|c|c|c|c|c|c|c|c|c|c|c|c|c|c|}
\hline \multirow[b]{2}{*}{ جمع } & \multicolumn{12}{|c|}{ نوع حادثد } & \multirow[b]{2}{*}{ سروه } \\
\hline & مسموميت & ماريزيدى عي و & ضربث & سوختخى & سقوط & ساير موارث & ختشونت & حيولات حيل & تصانفات & تصادف & خوندور سوار & مرفتنى برق & \\
\hline$\% \cdots$ & $\%$ & $\% \cdot \pi$ & $r$ & $\% 19$ & $\% 19$ & $\% V$ & $\% 1$ & $\% \cdot 4$ & $1 / \Delta$ & / & $\%$ & $\% \cdot \pi$ & كونكي \\
\hline$\% 1 \cdots$ & $\%$ & $\% \cdot r$ & $\% .01$ & 19 & $\% 1 \Delta$ & $\%$ & 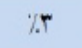 & $\% \cdot / 4$ & $1 / \Delta$ & $1 / 5$ & $\% F$ & $\% \cdot \mathbb{F}$ & نوجيولثي \\
\hline$\%$ & $\%$ & $\% \cdot \pi$ & 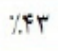 & $\%$ & \%tr & $\% \mathrm{~V}$ & $/ 4$ & $\% \cdot \pi$ & $1 / \Delta$ & $1 / 9$ & 19 & $\% \cdot 4$ & جوائي \\
\hline$\% \ldots$ & $\% 1$ & $\% \cdot \pi$ & 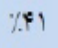 & $\% 1 \%$ & $\% 1 \mathrm{Y}$ & $\gamma \cdot \Lambda$ & $/ 4$ & $\% \cdot \pi$ & $1 / \Delta$ & $/ \Delta$ & $\% \Delta$ & $\% \cdot / 4$ & ميأسالى \\
\hline$\% 1$. & $\%$ & $\% \cdot \pi$ & $\% F$ & $\%$ & $\pi / T \Delta$ & $\%, 9$ & $\% 1$ & $\% \cdot / T$ & $1 \Delta$ & 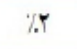 & $\% F$ & $\% \cdot \pi$ & سالمندى \\
\hline ث & $\%$ & $\% \cdot / \pi$ & 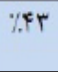 & $\%$ & $\% 19$ & $\% V$ & $\%$ & $\% \cdot / T$ & $1 \Delta$ & 19 & $\% \Delta$ & $\% \cdot / \pi$ & \\
\hline
\end{tabular}




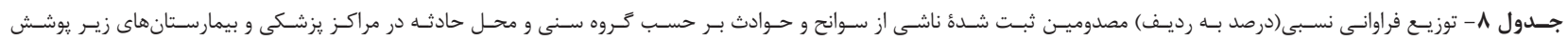

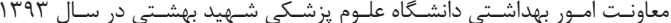

\begin{tabular}{|c|c|c|c|c|c|c|c|c|c|c|}
\hline \multirow[b]{2}{*}{ جم } & \multicolumn{9}{|c|}{ محل حادثد } & \multirow[b]{2}{*}{ سنى } \\
\hline & نامعلوم & منزل & آموزشيه & محل كار & كوجابه و & ساير & جزرّراه و & تفرزشى ورئى & عمومى & \\
\hline$\% 1 \cdots$ & $\% 1 \mathrm{~V}$ & $\gamma \mp \wedge$ & $\%$ & $\%$ & $\% 19$ & $\%$ & $\% r$ & $\%$ & $\%$ & كودكى \\
\hline$\% 1$ & $\% 11$ & $\% / r I$ & $1 / 0$ & $1 / 9$ & $\%$ YA & $\% / r$ & $\pi r$ & $\% 1$. & 1.0 & نوجوانى \\
\hline$\% \ldots$ & $\% 1 \mathrm{~V}$ & $\% / r$ & $\%$ & $\% 1 r$ & $\pi r$. & $\% r$ & $\% f$ & $1 / 0$ & $1 / 9$ & جواثى \\
\hline$\% 1 \ldots$ & $/ T_{1}$ & $\%$ & $\% \cdot$ & $7 / 1$ & $\% Y Y$ & $\% r$ & $\% r$ & $\%$ & $\%$ & مياتسالى \\
\hline$\% 1 \cdots$ & $\% 11$ & $\% \notin r$ & $\% \cdot$ & $7 / r$ & $\%$ ro & $\%$ & $\%$ & $\%$ & $\%$ & سالمندى \\
\hline $\begin{array}{l}\% \\
\%\end{array}$ & $\% 11$ & $\%$ \% & $\% 1$ & $\% 9$ & $\%$ YA & $\%$ & $\%$ & $\% . \Delta$ & $\% . \Delta$ & جمع \\
\hline
\end{tabular}

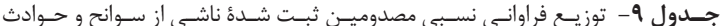

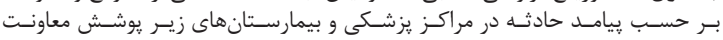

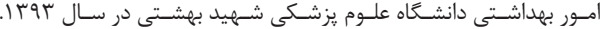

\begin{tabular}{|c|c|c|}
\hline فراوانى نسبى & تعداد & نتيجة حادثه \\
\hline$\% .99 / 1$ & |rIVG| & تحت درمان \\
\hline$\% \cdot / 1$ & 194 & فوت \\
\hline$\% \cdot / 1$ & it & معلوليت \\
\hline $\begin{array}{l}\% \\
\% \\
\end{array}$ & Ir। $94 V$ & $\varepsilon^{-a}$ \\
\hline
\end{tabular}

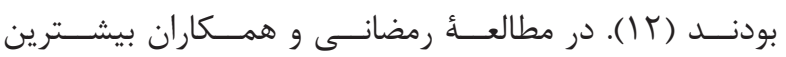

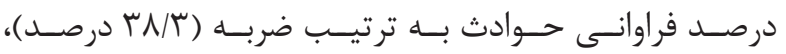

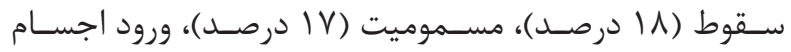

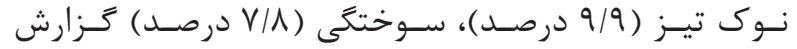

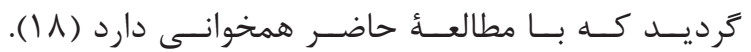

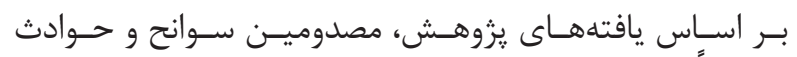

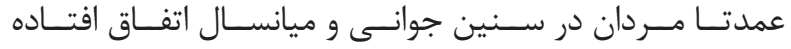

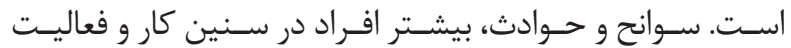

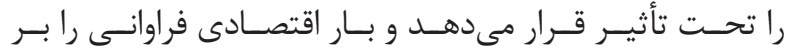

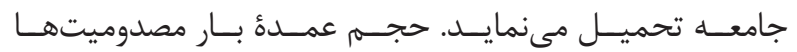

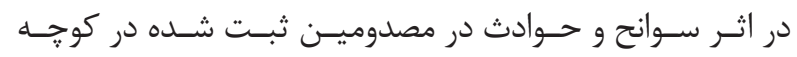

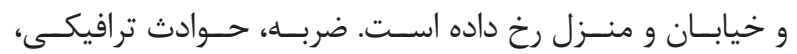

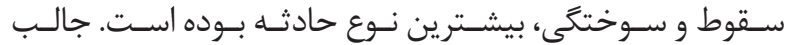

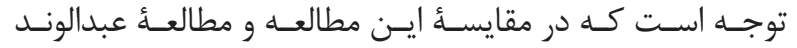

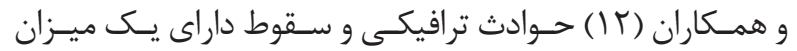

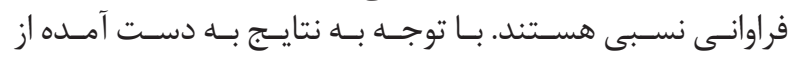

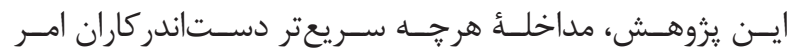

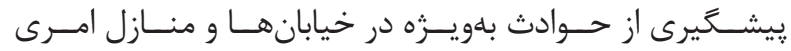

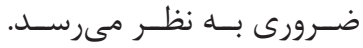

بحث و نتيجه كيرى

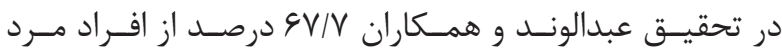

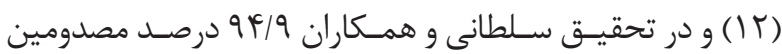

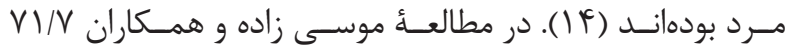

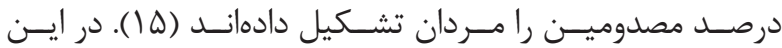

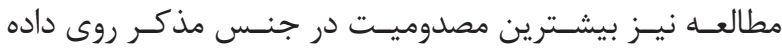

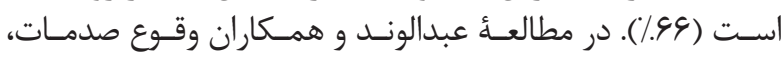

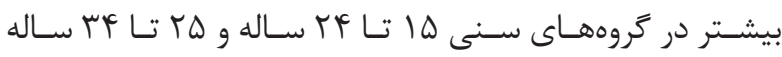

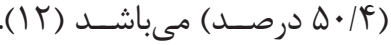

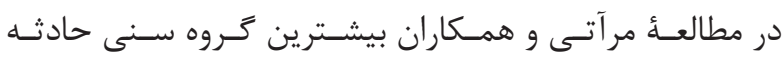

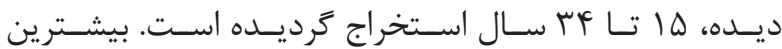

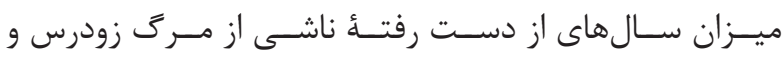

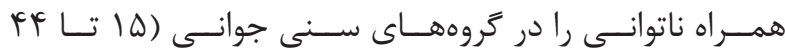

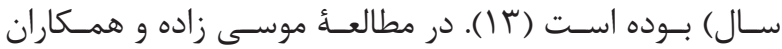

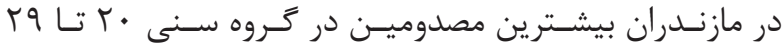

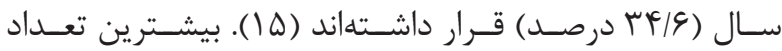

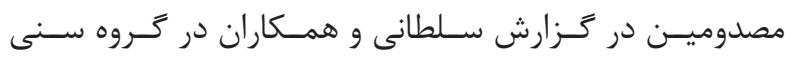

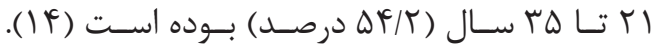

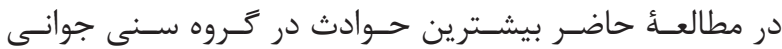

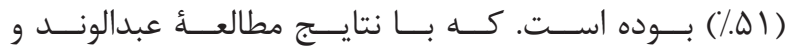

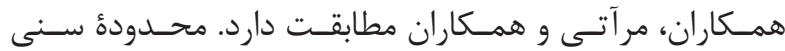

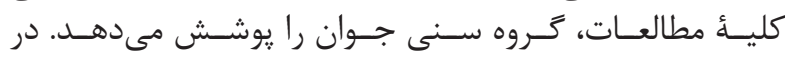

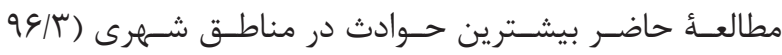

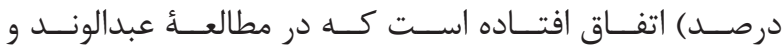

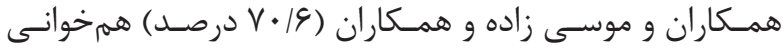

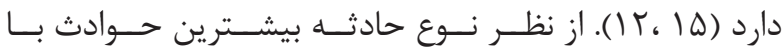

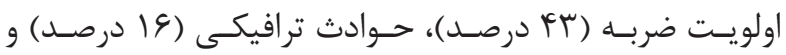

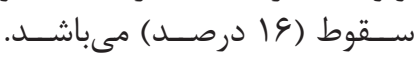

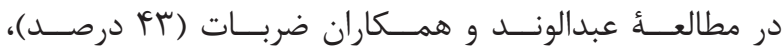

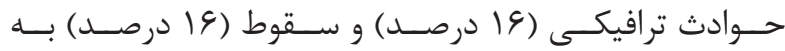

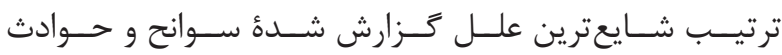




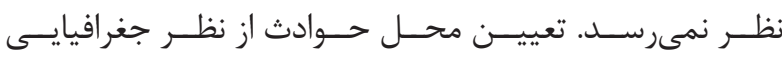

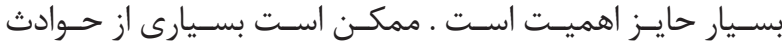

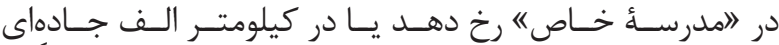

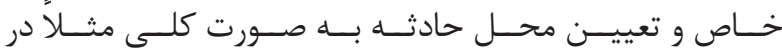

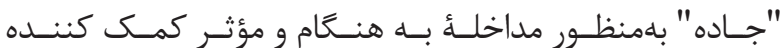

$$
\text { نمى باشـــد. }
$$

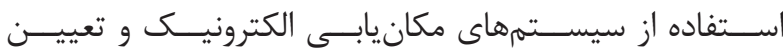

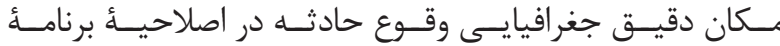

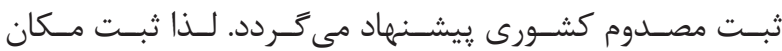

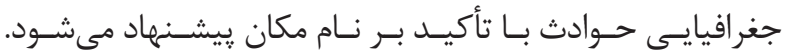

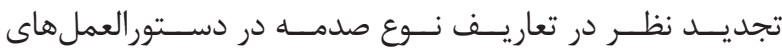

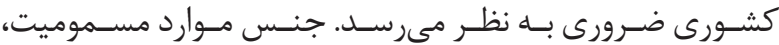

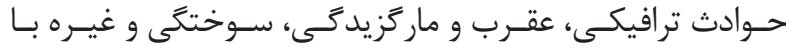

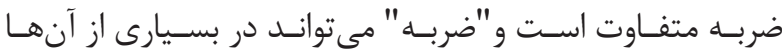

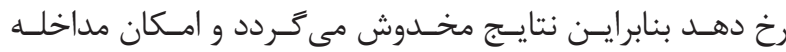

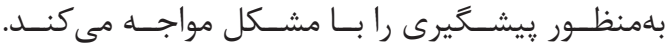

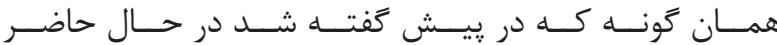

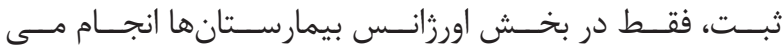

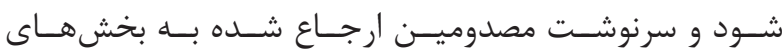

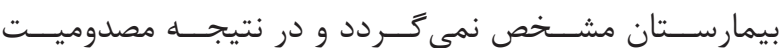

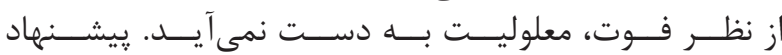

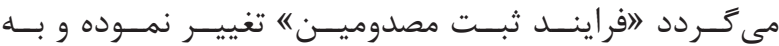

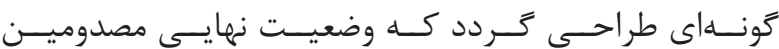

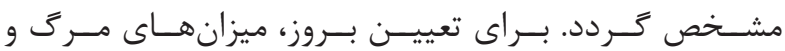

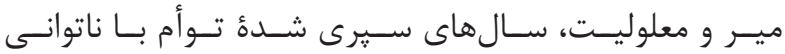

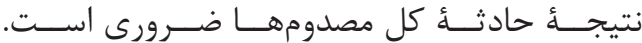

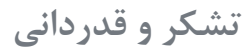

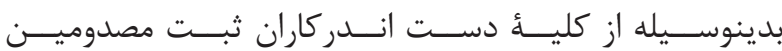

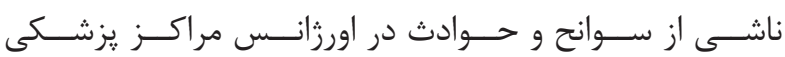

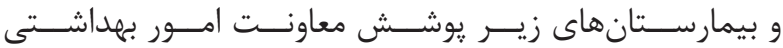

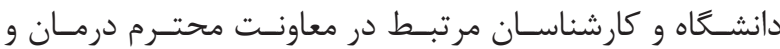

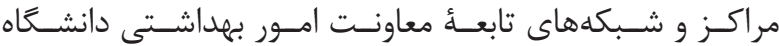
قدردانسى مى مَكـردد.

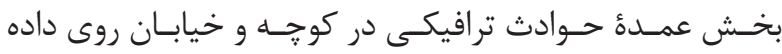

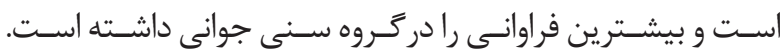

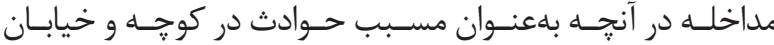

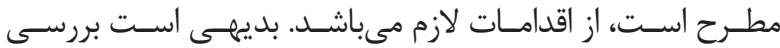

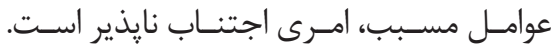

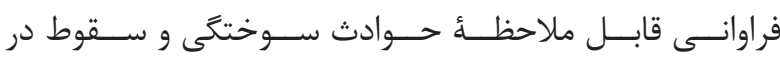

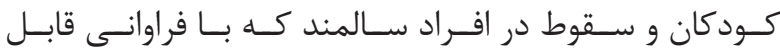

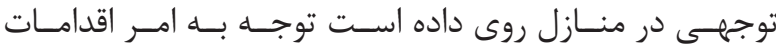

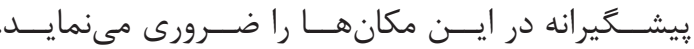

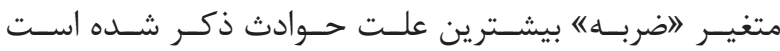

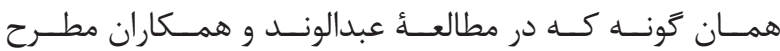

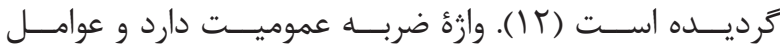

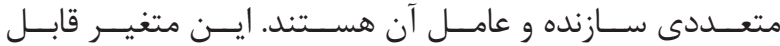

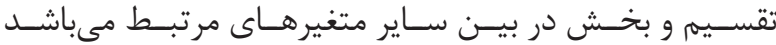

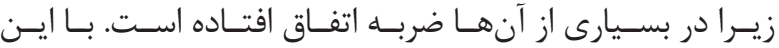

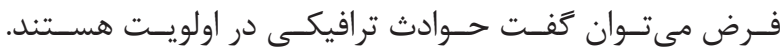

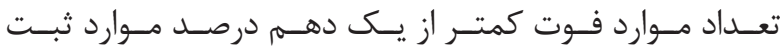

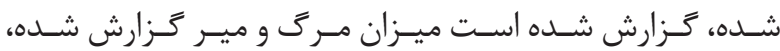

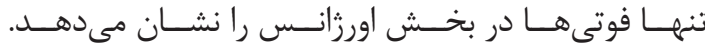

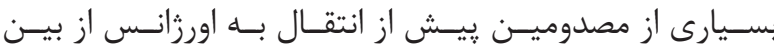

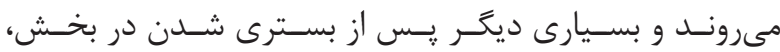

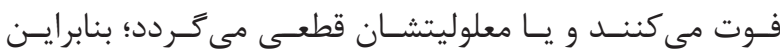

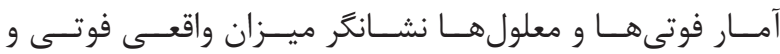

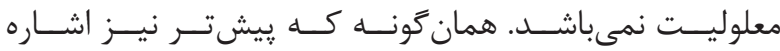

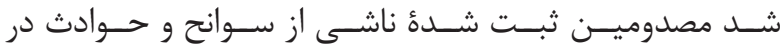

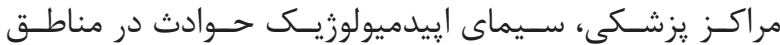

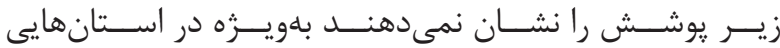

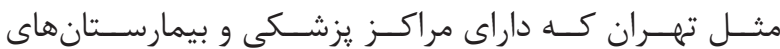

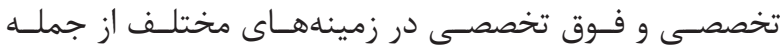

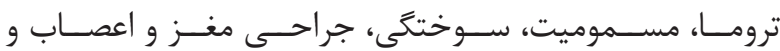

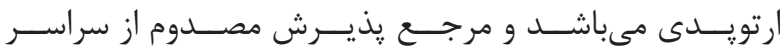

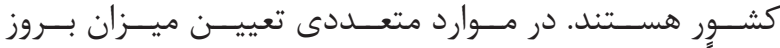

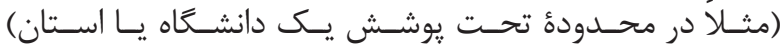

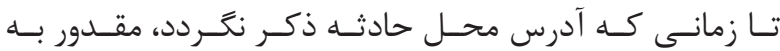


1. Soori H. Safety promotion and injury prevention. Intentional and Unintentional Injuries; Healthy Approach. 2013; 1(1): 1-11.

2. Neghab M, Rajaei Fard A, Habibi M, Choobineh A. Home accidents in rural and urban areas of Shiraz, 2000-02. East Mediterr Health J. 2006; 12(6): 824-33.

3. Neghab M, Rajaeefard A, Choobineh A. Home accidents in Shiraz during a 3-year period (2000-2002). Journal of Kermanshah University of Medical Sciences. 2008; 11(4): 428-40.

4. Mock CN, Boland E, Acheampong F, Adjei S. longterm injury related disability in Ghana. Disabil Rehabil. 2003; 25(13): 732-41.

5. Chandran A, Hyder AA, Peek-Asa C. The global burden of unintentional injuries and an agenda for progress. Epidemiol Rev. 2010; 32(1): 110-20.

6. Ansari-Moghaddam A, Martiniuk A, Mohammadi M, Rad M, Sargazi F, Sheykhzadeh K, et al. The pattern of injury and poisoning in South East Iran. BMC Int Health Hum Rights. 2012; 12: 17. doi: 10.1186/1472698X-12-17.

7. Peden M, McGee K, krug E. Injury: a leading cause of the global burden of disease 2000. Geneva: world Health organization. 2002.

8. Naghavi M, Abolhassani F, Pourmalek F, Lakeh M, Jafari N, Vaseghi S, et al. The burden of disease and injury in Iran 2003. Popul Health Metr. 2009; 7: 9. doi: 10.1186/1478-7954-7-9.

9. Peden M, scurfield R, sleet D, Mohan D, Hyder A, Jarawan E, et al. world report on road traffic injury prevention. Genova: world Health organization. 2004.

10. Souri H, Akbari M, Eini E, Zali AR, Naghavi M, Kordi Borujeni E. Epidemiology of nonfatal accidents in Iran. Journal of shahid beheshti school of nursing and midwifery. 2008; 18(60): 45-50.

11. Tavakoli Kashani A, Shariat-Mohaymany A, Ranjbari A. Analysis of factors associated with traffic injury severity on rural roads in Iran. J Inj Violence Res. 2012; 4(1): 36-41.

12. Abdolvand $\mathrm{M}$, Monfared $\mathrm{AB}$, Khodakarim S, Farsar AR, Golmohammadi A, Safaei A. Evaluation of accidents and incidents at injury registered in medical centers affiliated to shahid beheshti university of medical sciences (2012-2013). Safety Promotion and Injury Prevention. 2014; 2(1): 65-72.

13. Marati MR. The burden of road traffic injuries in Isfahan, Iran in 2010. Journal of Kerman University of Medical Sciences. 2013; 20(5): 505-19.

14. Soltani GH, Ahmadi B, Pourreza A, Rahimi A. Investigating prevalence of deaths from traffic accidents and factors associated with it in Yazd in 2009. Journal of Shahid Sadoughi University of Medical Sciences. 2014; 21(6): 831-9.

15. Moosazadeh M, Nasehi MM, mirzajani M, Bahrami MA. Epidemiological study of traumatic injuries in emergency departments of Mazandaran hospitals, 2010. Journal of Mazandaran University of Medical Sciences. 2013; 23(98): 144-54.

16. Bramer GR. International statistical classification of diseases and related health problems. $2^{\text {nd }}$ ed. Genova: world Health organization. 1988; p. 32-6.

17. ghadiri M, hadadi M. Guidline of injured registery duo to accident. In: disaster cfdma. Tehran: ministry of health. 2012; p. 1-3.

18. Ramazani AB, Izad Khah MH, Gholeenejad $B$, Amirabadizadeh $H$. Epidemiologic study and relationship factors of home injuries in clienteles to Birjand, s hospital in 2004. Journal of Rostamineh. 2011; 2(3): 71-9. 